\title{
Remote Monitoring System for a Photovoltaic Power Substation
}

\author{
http://dx.doi.org/10.3991/ijoe.v11i1.3923 \\ Wang Wenxing \\ North China University of Water Resources and Electric Power, Zheng Zhou, P.R. China
}

\begin{abstract}
After briefly analyzing the structure of a photovoltaic power substation monitoring system, a positioning system roadway based on blue tooth was put forward to connect with already existing communication and monitoring networks. A separate monitoring system for the environment is integrated with the former comprehensive automation system to create a unique system. It is a new step of substation automation. The system architecture, software, hardware, and necessary factions of this system are introduced in detail. In photovoltaic power substation tunnels, monitoring identity code terminal stations are set along the power line information to collect personnel information data by receiving wireless signals.
\end{abstract}

Index Terms-Bluetooth, Photovoltaic power substation, sensor networks, computerized monitoring, remote sensing

\section{INTRODUCTION}

Energy is the main basis for human survival, life and development. Currently over 85 percent of the total global use of energy is fossil fuels, including coal, oil and natural gas. Fossil fuel reserves are limited, as coal, oil and natural gas are being depleted, which will cause a serious energy shortage crisis and an inability to maintain production over many decades. With the advent of renewable energy, many countries are paying attention to solar as a clean energy solution but do not evaluate its failure characteristics.

Exploitation of solar, with its unique advantages, is bound to lead to a shift in the structure of energy in the world away from fossil fuels and toward renewable sources.

Abundant solar energy resources are widely distributed and offer the most promising renewable energy. As problems of the global energy shortage and environmental pollution become increasingly prominent, solar photovoltaic energy will occupy an important consumption posi- tion in the world, not only to replace part of the conventional energy sources but to become the main body of the world's energy supply.

\section{Photovoltaic Systems}

Figure 1 is a block diagram of the photovoltaic (PV) power generation system. After the inverter converts DC to AC grid requirements directly into electrical energy through the large power substation, the system generated by the PV array photovoltaic cell assembly is supplied in addition to the load generated by the outer remaining electricity directly to the grid. On weaker light rainy days or at night, photovoltaic modules generate electricity or no electricity generated by the power can not supply just load and can requirement from net.

The connection limits formed by the bypass of a double-bus in the application of the substation is common, but having each segment on each set form a bypass bus section circuit breaker is not as common. A bypass breaker cannot be both at the same time or always be in good condition. It will also need repair and maintenance. Repairs should be done when one is in a standby state, so reliability is higher than a bypass bus without a fragment and having only set one breaker. The type of switching station is an outdoor, single phase medium-sized layout [1]. The power structure is fixed, lacks flexibility, and all of the intelligent devices lack overall coordination. Bluetooth wireless communication technology can solve the above referenced problem [2]. Most of the current substation models have centralized control; the centralized control center generally uses the unattended mode, does not have a wireless alarm, and is necessary to improve the communication capacity of the station's automation system. The remote monitoring system uses three tandem structures: the control center, monitoring unit and monitoring sensor [3]. More and more enterprise applications use an RFID technique [4].

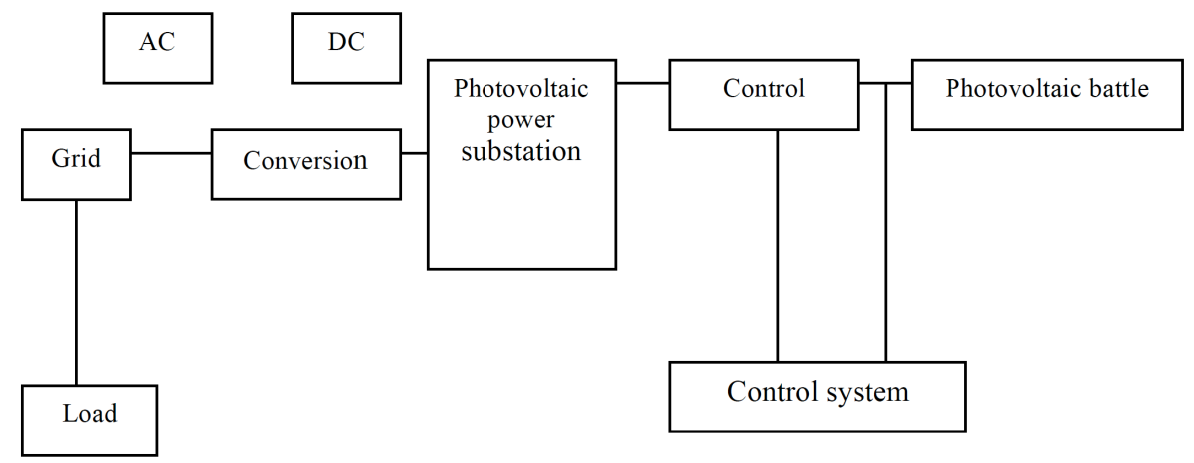

Figure 1. Block diagram of grid 


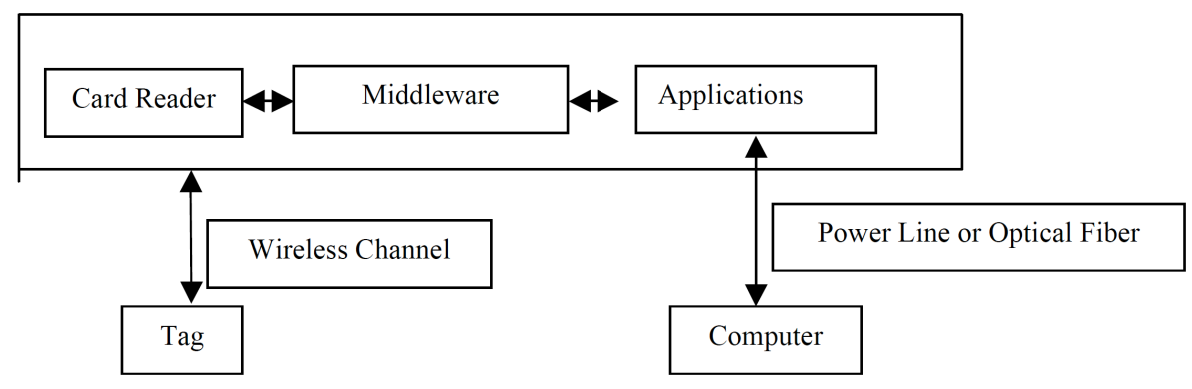

Figure 2. System Structure

\section{SYSTEM ARCHITECTURE}

The technology based on Bluetooth can effectively simplify the communication between mobile devices in a substation and successfully streamline the devices and equipment and communication with the Internet, so communication between devices and the Internet data is quicker and more efficient in order to widen the road substation information and communication. The technology based on Bluetooth is actually a short-range wireless communication technology. Bluetooth specification microwave bands have a $1 \mathrm{M}$ bytes per second transfer rate. The maximum transmission distance is 10 meters but can be up to 100 meters by increasing the transmitting power. This distance is suitable for measurement of photovoltaic devices. Bluetooth is open globally, has good compatibility on a global scale, and has a low-cost to get Bluetooth network.

It has wireless, open, low power characteristics and is one of the non-contact automatic identification technologies.

It uses radio frequency technology and magnetic coupling characteristics to achieve the automatic identification of objects.

The reader sends signals through an antenna; radio frequency tags obtained by sensing the current energy will be stored in the chip containing the encrypted information signal the substation equipment. The reader receives the information and the decoded information extraction equipment from the information processing system, the completion of the default function, and automatic identification.

The distance between the central control room and the equipment is less than $100 \mathrm{~m}$. Currently a high-power Bluetooth chip can achieve $100 \mathrm{~m}$ coverage, fully meeting the actual application.

In the hydropower substation tunnel, monitoring the identity code terminal stations are set along the power line information to collect personnel information data by receiving wireless signals. The system is tested in the corridors. Collected data from various stations via the cable are sent to the transit center set up at the bottom of the field or at the central substation gateway. They are transmitted by the power line or optical fiber ground hosts. The host from the ground completes the data analysis, processing and storage. Therefore, the current Bluetooth, although just started, has caused great concern in mobile communication research.[6]. It achieves the lowest power consumption and reduces cubage. The problems are higher development costs and higher risks [7]. Ground host stations are connected with each to complete the system. In this paper, power line is used as signal transmission medium in the main roadway, and spread spectrum is used as immunity impacts. In a communication system, a key performance indicator for the user is the throughput [8]. In order to reduce the cost, signal transmission in wireless and wire will realize compatibility based on certain foundations. It is the trend [9]. The Bluetooth module is connected with a USB attended mode, takes the Bluetooth module as a USB slave device, and hosts the communication; the maximum transmission speed can reach $12 \mathrm{Mbit} / \mathrm{s}$ [10].

The hardware of the single chip mainly consists of the core single chip, SRAM, keyboard, LCD, wireless module and components. The system structure architecture is shown as Figure 2.

The core single chip adopts internal storage capacity, resource-rich chips various interfaces to meet the actual needs of the scene. SRAM is the internal memory supplement used for data caching and storage. The core single chip via a wireless network connects with the substation monitoring system to achieve real-time data communication. Bluetooth working frequency is $2.4 \mathrm{GHz}$ [11], ISM (industry, science, and medical frequency band), without a license. Most countries use frequency 79 [12], Keyboard and LCD modules are interactive and offer easy operator use. The information collection system uses a wireless, long-distance cable transmission mode. Code transmitter remote terminals are provided by staffing, make the signal a carrier of information, and send out its own personnel number through radio signals.

\section{SYSTEM HARDWARE}

Due to internal resources and pins a Bluetooth chip is limited; therefore, data acquisition and control from the front end is completed by $51 \mathrm{MCM}$, the serial connection width of a serial port of a Bluetooth chip. The signal is transformed into digital information via a Bluetooth, does not need to change the original program, and is sent to the control center.

The high voltage and high current from the transformers and circuit breakers are changed into safe voltage for collection $(0 \sim 5 \mathrm{~V})$ by the voltage transformer or current transformer, which are transformed by a single-chip microcomputer through the A/D. After computing the corresponding, it is sent into the Bluetooth chip.

After the Bluetooth chip packages the data, the signal is sent to the control room through a wireless or wired communication channel. The system hardware architecture is shown as Figure 3.

The traditional wired correspondence equipment can satisfy the information transmission request of the stationary dot consumer. Due to the current Bluetooth transmission limitations of distance, according to the White Paper 
Bluetooth HCI protocol, HCI is the host and host controller interface. The host refers to the original equipment circuit; the controller is a Bluetooth module, according to the different application programming. The Data Acquisition Block Diagram is shown as Fig.4.

Meanwhile, the terminals will also receive commands through Bluetooth chips or cable channels from the control room and send the commands to the micro controller. After receiving the analysis, the microcomputer isolation switches are controlled to make corresponding moves. Bluetooth nets of network mode are only suitable for small and medium-sized substations; if they are used in large substations, they need to be a more complex network structure.

\section{SOFTWARE DESIGN}

Bluetooth protocol procedures to the underlying object code format are stored in the baseband processor, and the host processor supports the upper layer protocols of the original program.

In a Bluetooth-enabled system, procedures are obtained from the original upper layer protocol and are integrated into the host application program. The host is connected with the underlying protocol by the HCI (Host Controller Interface). During the Bluetooth system design, RF and baseband technology outsourcing as well as LM(Lan Manager), HCI, the underlying protocol, HCI drive and other upper layer protocol software such as L2CAP(Logical Link Control and Adaptation Protocol), SDP (Service Design Package), RFCOMM, etc. were required to be installed in the host application software to support the Bluetooth. In addition, some embedded Bluetooth protocol and the underlying protocols are integrated in the upper single Bluetooth chip.

Front-end data acquisition is placed in a high-voltage door; the information sent system block diagram is shown as Fig.5. Using a handheld wireless access device can achieve wireless access within the station. The signal emission control center can be set in a high pressure chamber and control room, can cover the entire substation, and can achieve wireless signals with a seamless connection. In the transformer substation, the functions of the devices in the control room are measured. It collects time of data collection, complete measurement, control and protection function, a large number of data processing in scheduling end completed, $1 \mathrm{Mb} / \mathrm{s}$ rate of Bluetooth can meet the requirements.

\section{CONCLUSION}

The photovoltaic power system based on a Bluetooth system is a further development of substation automation technology. The system used in the substation can realize remote control, monitoring, supervision, and other functions to improve the communication capabilities. The system will also facilitate maintenance and troubleshooting, improve efficiency, safety and the economic benefits of the power grid.

\section{REFERENCES}

[1] PENG Xiao-he, ZOU Gong-sheng. Problem and Solution of 200 $\mathrm{kV}$ System of Erijiang Hydropower Plant in Gezhouba. Central China Electric Power. 2003,3:66-67 [1 2] J. Misic, V. B. Misic. Bridges of Bluetooth County: Toplogies, Scheduling, and Performance [J] .IEEE Journal on Selected Areas in Communications, 2003,21:240-258

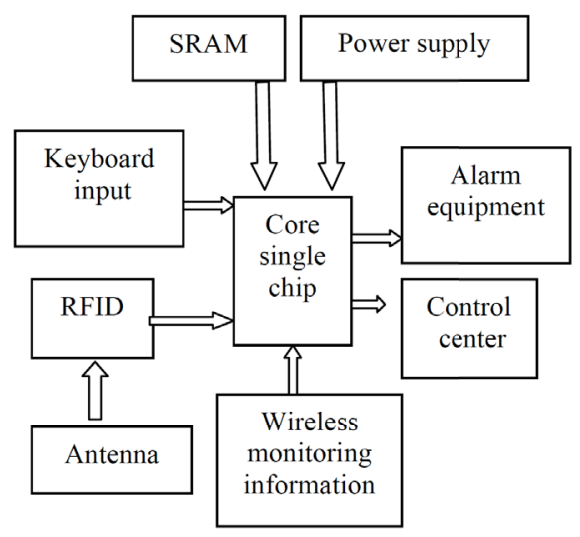

Figure 3. Hardware functional block diagram

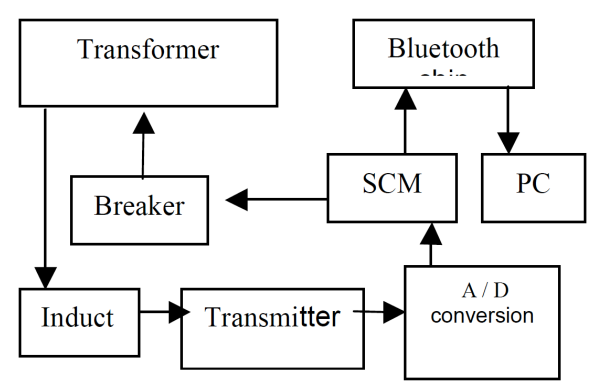

Figure 4. Data acquisition block diagram

[2] BILSTRUP U, WIBERG P A. Bluetooth inIndustrial Environment: Proceedings of the 11th IEEE International Workshop Factory Communi-cation Systems [C]. Portugal, 2000: 239 246

[3] Wang Wenxing. Research on Remote Condition Monitoring System in Coal Mine. International Journal of online Engineering. 2014,(1):37

[4] Kong Xiangsheng. RFID Event Analysis Based on Complex Event Processing. International Journal of online Engineering. 2014,(1): 5

[5] Ning Chen,Xiao-ping. SongSchema Design of communication module in Highway Monitoring System: A Case Study. International Journal of online Engineering. 2014,(1): 10

[6] John Newbury, Communication Requirements and Standards for Low Voltage Mains Signaling, IEEE Transaction Power Delivery, Vol.13, Issue 1, 1998 , pp.46-53

[7] VRANA G. Untangling IrDA and Bluetooth [J]. Electronics Engineering, 2002,35(2):16 18

[8] Xie Xiren. Computer Network, Electronic Industry Press, 2002 pp. 53

[9] DAI Zhen-yu. Comparison Between Wire and Wireless Communication, CHINA CABLE TELEVISION, Vol. 4, 2003

[10] Cao Jie, Bai Junli. The Design of Bluetooth Ad-Hoc Network for the Multi-entertainment Robot System. Electrical Automation2001, 4(32) 29-30

[11] He Guoquan Li Yaozhong the Control of Bluetooth Module Based on MCU in HCI Layer. Technology Square 2005.1 43-46

[12] WANG Xiao-yan,WANG Hao,KE Zhi-wu, Design and Implementation of Remote Controlling Systembased on Bluetooth

[13] HE Qing - hua. Design and Implementation of Remote Engineering Equipment Monitoring System. JOURNAL OF ZHENGZHOU UNIVERSITY (ENGINEERING SCIENCE), 2009,30(2): 66-70

\section{AUTHOR}

Wang Wenxing is with North China University of Water Resources and Electric Power, Zheng Zhou, P.R. China, 450011.

Submitted 31 May 2014. Published as resubmitted by the author 25 January 2015 . 